07,12

\title{
Механизм влияния поли- и нанокристалличности на параметры кривых псевдоупругой деформации сплавов с эффектом памяти формы
}

\author{
(C) Г.А. Малыгин \\ Физико-технический институт им. А.Ф. Иофффе РАН, \\ Санкт-Петербург, Россия \\ E-mail: malygin.ga@mail.ioffe.ru
}

(Поступила в Редакцию 10 мая 2018 г.)

\begin{abstract}
В рамках теории размытых мартенситных переходов проанализировано влияние поликристалличности и нанокристалличности на параметры кривых псевдоупругой деформации сплавов с эффектом памяти формы (ЭПФ). С учетом нормальной плотности распределения зерен по их ориентациям и лог-нормальной по размерам зерен $d$ впервые удалось установить функциональную и количественную зависимость коэффициента деформационного (мартенситного) упрочнения сплава с ЭФП от размера зерен $d$ и ориентационного фактора $M, d \sigma / d \varepsilon \sim 1 / M d^{2}$. При сравнении найденной зависимости с имеющимися в литературе данными для нанокристаллического сплава TiNi c размером нанозерен в диапазоне 8-90 nm получено хорошее согласие теории и эксперимента.
\end{abstract}

DOI: $10.21883 /$ FTT.2018.10.46529.123

\section{1. Введение}

Структура сплавов с эффектом памяти формы (ЭПФ) оказывает большое влияние на параметры кривых их псевдоупругой деформации и, следовательно, на функциональные свойства сплавов, определяющие их применение в различных устройствах. Известно, например, что обратимая мартенситная деформация у поликристаллических образцов сплава существенно меньше, чем у их монокристаллических аналогов [1]. С другой стороны, напряжение начала псевдоупругой деформации у поликристаллов выше, чем у монокристаллов. Оба эти параметра (обратимая деформация и напряжение начала мартенситной текучести) определяют применение сплава в том или ином техническом (сенсорном или силовом) устройстве. Наличие в сплаве с ЭПФ дисперсных наночастиц также является важными фактором изменения и улучшения его функциональных свойств [2].

В последнее время внимание исследователей привлечено к изучению свойств ЭПФ сплавов в их суперполикристаллическом (нанокристаллическом) состоянии [3-6], а также наноразмерных (в одном или двух направлениях) монокристаллов этих сплавов [7]. Эксперименты показали, что с уменьшением размера нанозерен в поликристалле или поперечного размера нанокристалла напряжение начала их мартенситной деформации существенно возрастает, увеличивается при этом также их коэффициент деформационного (мартенситного) упрочнения [3]. Кроме того, было обнаружено, что начиная с некоторого критического размера нанозерен $[3,6]$ или размера нанокристалла, критическая температура прямого мартенситного перехода обращается в нуль и переход оказывается заблокированным.
Чтобы понять и объяснить всю эту совокупность экспериментальных фактов теория мартенситных превращений должна быть чувствительна к структуре сплава на мезоуровне. Такой структурно-чувствительной теорией является теория размытых мартенситных переходов (МРП) [8]. Она уже использовалась ранее при анализе размерных эффектов в сплавах с ЭПФ $[9,10]$. В настоящей работе она применена для анализа и моделирования влияния поли- и нанокристалличности на кинетику мартенситного перехода в сплаве TiNi. B [3] методом молекулярной динамики (МД) получены кривые псевдоупругой деформации этого сплава при варьировании размера нанозерен в диапазоне от 8 до $30 \mathrm{~nm}$. В указанной работе приведены также результаты реальных экспериментов других исследователей [4] со сплавом TiNi при варьировании размера зерен в диапазоне 20-90 nm. Таким образом, эти работы охватывают весь диапазон нанокристалличности сплава. Целью настоящей работы является анализ этих данных в рамках теории РМП и установление функциональной зависимости параметров кривых псевдоупругой деформации сплава TiNi от размера нанозерен, а также от ориентации их габитусных плоскостей относительно направления действующего напряжения.

\section{2. Поликристалличность и нанокристалличность}

Согласно теории РМП при одностадийном мартенситном переходе объемная доля мартенсита в кристалле зависит от температуры $T$ и напряжения $\sigma$ согласно соотношению [8]

$$
\varphi_{M}=\frac{1}{1+\exp (\Delta U / k T)},
$$


где $\Delta U=\omega \Delta u-$ изменение свободной энергии сплава при образовании в нем зародыша новой фазы объемом $\omega$

$$
\Delta u=q \frac{T-T_{c}}{T_{c}}-\varepsilon_{m}(\sigma \mp \Delta \sigma),
$$

$\Delta u$ - объемная плотность свободной энергия сплава, $q$ - теплота (энтальпия) перехода, $T_{c}-$ характеристическая температура превращения, $k-$ постоянная Больцмана, $\varepsilon_{m}$ - деформация превращения, $2 \Delta \sigma-$ гистерезис мартенситного перехода под напряжением. Уравнение (1) описывает равновесие мартенситной $\varphi_{M}$ и аустенитной $\left(\varphi_{A}=1-\varphi_{M}\right)$ фаз в зависимости от температуры и напряжения.

2.1. Поликристалличность. По сравнению с монокристаллами поликристалличность материала имеет две особенности. Во-первых, это наличие зерен, различно ориентированных по отношению к направлению действия напряжения, во-вторых, это размер зерен $d$ и его влияние на напряжение течения. Обе эти особенности присущи также и мартенситной деформации. Здесь имеет значение ориентация плоскости габитуса относительно приложенного к поликристаллу напряжения $\sigma$, определяющая величину касательного напряжения $\tau=m_{s} \sigma$ и деформацию мартенситного сдвига $\gamma=\varepsilon / m_{S}$ в плоскости габитуса, где $m_{S}$ - ориентационный коэффициент, аналог фактора Шмида при пластической деформации кристаллов.

Рассмотрим вначале, как в рамках теории РМП ориентация зерен влияет на параметры кривой псевдоупругой деформации сплава с ЭПФ по сравнению с монокристаллами. При одноосном сжатии или растяжении монокристалла для его мартенситной деформации $\varepsilon_{M}=\varepsilon_{m} \varphi_{M}$ согласно (1) имеем соотношение

$$
\varepsilon_{M}(T, \sigma)=\frac{\varepsilon_{m}}{1+\exp \left[\left(\frac{\omega q}{k T}\right)\left(\frac{T}{T_{c}}-1-\frac{\sigma \mp \Delta \sigma}{\sigma_{m}}\right)\right]},
$$

где $\sigma_{m}=q / \varepsilon_{m}, \varepsilon_{m}=m_{S}^{\max } \xi-$ деформация кристалла при максимальной величине ориентационного коэффициента $m_{S}, \xi$ - деформация решетки кристалла при ее структурной перестройке. Ориентационный коэффициент изменяется в ГЦК кристаллах от минимального значения 0 до максимального $\approx 0.5$. Для последующего плавного перехода от деформации монокристаллов к деформации поликристаллов удобно ввести относительный ориентационный параметр $m=m_{S} / m_{S}^{\max }$, изменяющийся в пределах $0-1$. В таком случае с учетом упругой деформации кристалла и нагружающего устройства $\sigma / K$ для нахождения зависимости напряжения от полной деформации кристалла $\varepsilon$ и ориентационного параметра $m$ имеем уравнение

$$
\varepsilon=\frac{\sigma}{K}+\varepsilon_{M}(T, \sigma, m),
$$

где $K-$ модуль упругости системы кристаллнагружающее устройство. Уравнение (3a) содержит зависимость $\sigma(\varepsilon)$ в неявном виде. Для численного реше- ния уравнения (3a) удобно записать его в развернутом виде и в безразмерных переменных и параметрах

$$
\bar{\varepsilon}=\frac{\bar{\sigma}}{\bar{K}}+\frac{m}{1+\exp [\bar{\omega}(\bar{T}-1-(\bar{\sigma} \mp \Delta \bar{\sigma}))]},
$$

где $\quad \bar{\varepsilon}=\varepsilon / \varepsilon_{m}, \quad \bar{\sigma}=\sigma / \sigma_{m}, \quad \bar{K}=K / \sigma_{m} \varepsilon_{m}, \quad \bar{\omega}=\omega q / k T$, $\bar{T}=T / T_{c}, \Delta \bar{\sigma}=\Delta \sigma / \sigma_{m}$. На рис. $1, a$ приведены результаты решения уравнения (3b) для трех значений параметра $m$ при следующих значениях остальных параметров: $\bar{K}=1, \bar{\omega}=100, \bar{T}=1.1,2 \Delta \bar{\sigma}=0.05$. Видно, что по мере снижения величины параметра $m$ от наиболее благоприятного значения $m=1$ до менее благоприятного $m=0.1$, параметры кривой псевдоупругой деформации существенно изменяются. С уменьшением возрастает напряжение начала мартенситной деформации, а также наклон кривой $\bar{\sigma}-\bar{\varepsilon}$ в средней ее части, т.е., безразмерный коэффициент деформационного (мартенситного) упрочнения $\bar{\theta}=d \bar{\sigma} / d \bar{\varepsilon}$,

$$
\bar{\theta}=\frac{1}{\bar{K}^{-1}+m \bar{\omega} \varphi_{M}\left(1-\varphi_{M}\right)},
$$

где $\varphi_{M}=\varepsilon / \varepsilon_{m}$. Коэффициент $\bar{\theta}$ достигает минимального значения $\bar{\theta}_{m}$ при $\varphi_{M}=1 / 2$. Далее, поскольку $\bar{K}^{-1} \ll \bar{\omega}$, то, переходя к размерным переменным и параметрам, получаем следующую зависимость коэффициента деформационного упрочнения $\theta_{m}$ от ориентационного параметра $m$

$$
\theta_{m}=\frac{4 k T}{m \omega \varepsilon_{m}^{2}} \sim m^{-1}
$$

На рис. $1, b$ в качестве иллюстрации приведены также результаты решения уравнения (3b) при температуре чуть выше критической, $T=1.02 T_{c}$, и значениях остальных параметров, тех же что и для кривых $\sigma / \sigma_{m}-\varepsilon / \varepsilon_{m}$ на рис. $1, a$. Видно, что остаточная деформация после разгрузки кристалла (деформация памяти формы) существенно снижается при уменьшении величины параметpa $m$.

В поликристалле существует разнообразие ориентировок зерен, оно может изменяться от полностью хаотического до концентрации их вблизи некоторой определенной ориентировки (при наличии в поликристалле текстуры). Это зависит от плотности распределения ориентировок и от дисперсии этого распределения. В качестве примера рассмотрим нормальное (гауссово) распределение плотности ориентационного параметра $m$

$$
f(m, \mu, s)=\frac{1}{s \sqrt{2 \pi}} \exp \left[-\frac{1}{2}\left(\frac{m-\mu}{s}\right)^{2}\right],
$$

где $\mu$ - некоторая определенная его величина, $s-$ дисперсия распределения. На рис. 2, $а$ показано распределение плотности параметра $m$ около наиболее благоприятной в смысле ориентации величине $\mu=1$ и трех значениях дисперсии распределения $s=0.1,0.3$ и 1.0. Видно, что при малой величине дисперсии основной 

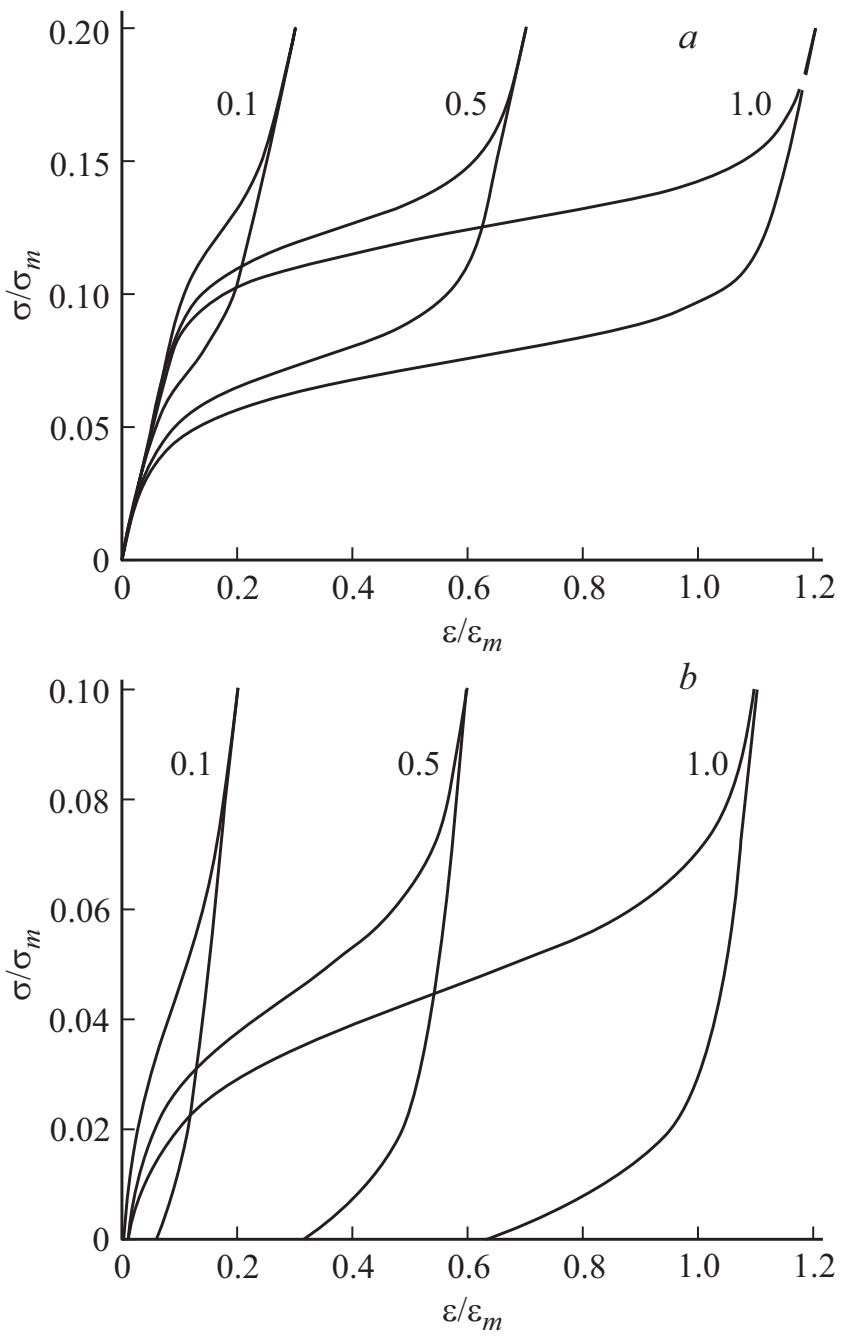

Рис. 1. Кривые псевдопругой деформации монокристалла сплава с ЭПФ при температурах $T=1.1 T_{c}$ (a) и $T=1.02 T_{c}(b)$ согласно уравнениям (3) при трех различных значениях ориентационного параметра $m$ (цифры у кривых).

вклад в плотность распределения вносят наиболее благоприятные ориентировки, но по мере роста дисперсии начинают вносить вклад и менее благоприятные. При $s \geq 1$ все ориентировки в интервале $m=0 \div 1$ вносят практически одинаковый вклад в плотность распределения $m$. Усредняя уравнение (3b) с помощью (5) по параметру $m$, получаем уравнение

$$
\bar{\varepsilon}=\frac{\bar{\sigma}}{\bar{K}}+\frac{M(s)}{1+\exp [\bar{\omega}(\bar{T}-1-(\bar{\sigma} \mp \Delta \bar{\sigma}))]},
$$

где

$$
M(s)=\int_{0}^{1} m f(m, 1, s) d m,
$$

- ориентационный фактор поликристалла, аналог фактора Тейлора $M_{T}$ при пластической деформации поликристалла, $M=1 / M_{T}$. На рис. $2, b$ показана его зависимость от $s$, а на рис. 3 приведены согласно (6а) кривые деформации $\sigma / \sigma_{m}-\varepsilon / \varepsilon_{m}$ при температуре $T=1.1 T_{c}$ и двух значениях $M(0.1)=0.46$ и $M(1)=0.18$. Пунктиром на рис. 3 обозначена кривая деформации монокристалла сплава при $m=1$ (см. рис. $1, a$ ). Видно, что по сравнению с монокристаллом поликристалличность сплава в $M(s)$ раз снижает деформацию мартенситного превращения сплава и в $1 / M(s)$ раз увеличивает коэффициент его деформационного упрочнения,

$$
\theta_{m}=\frac{4 k T}{\omega M(s) \varepsilon_{m}^{2}}
$$

Проанализируем теперь, как температура, ориентационный параметр $m$ и дисперсия ориентаций зерен $s$ влияют на остаточную деформацию (деформацию памяти формы $\varepsilon_{S M}$ ) при разгрузке деформируемого образца. Полагая в уравнениях (3b) и (6a) $\sigma=0$, получаем зависимость остаточной деформации от температуры и
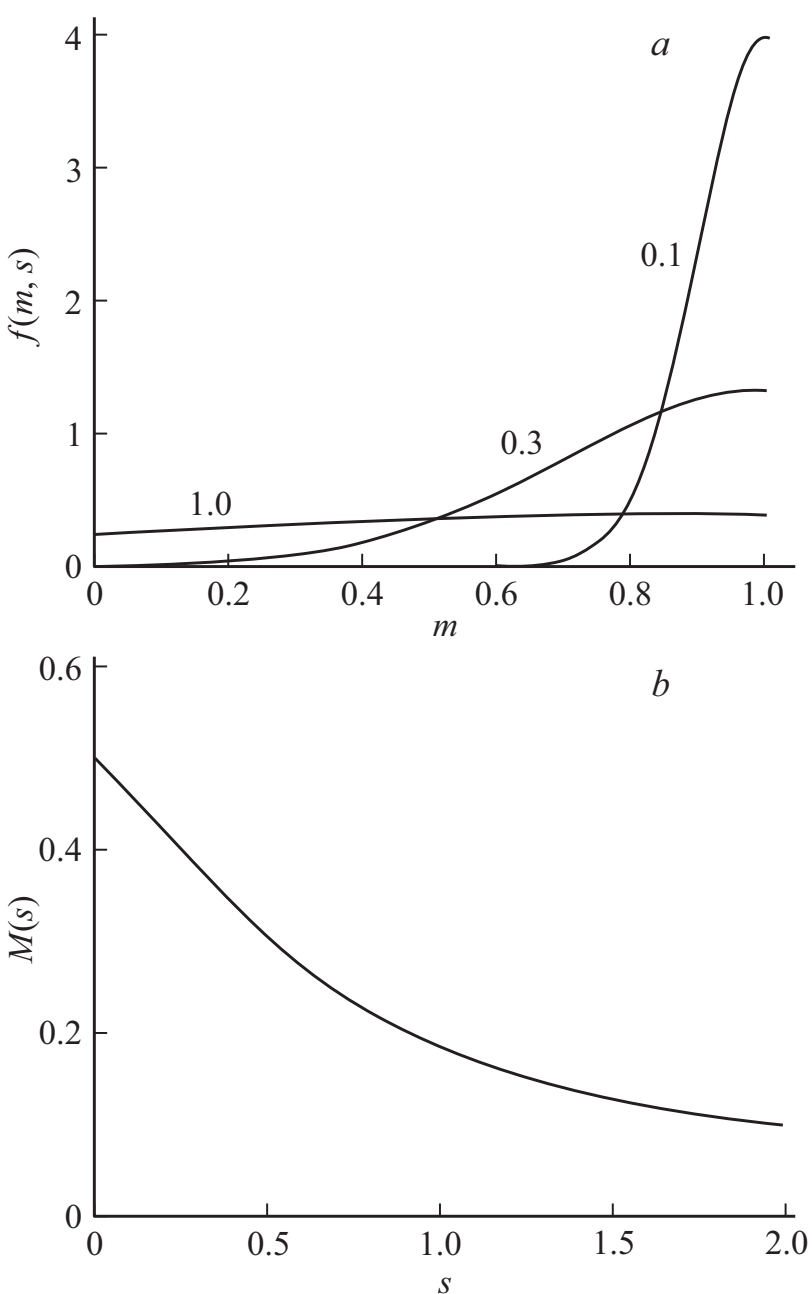

Рис. 2. Кривые распределения величины ориентационного параметра $m$ зерен в поликристалле согласно уравнению (5) при трех различных значениях величины дисперсии $s$ (цифры у кривых) $(a)$, и $(b)$ зависимость ориентационного фактора $M$ поликристалла согласно (6b) от величины дисперсии зерен по ориентациям. 


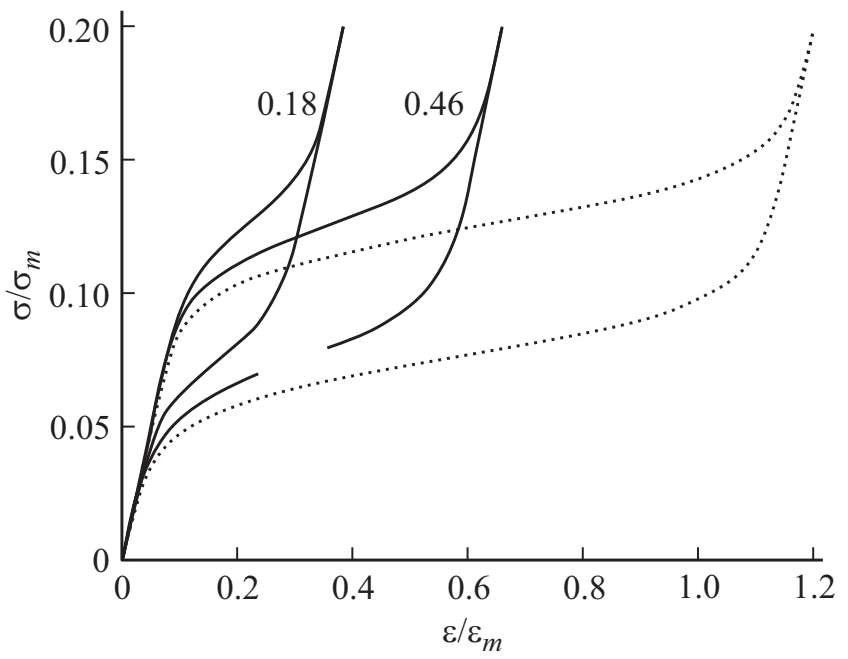

Рис. 3. Кривые псевдоупругой деформации поликристалла сплава с ЭПФ согласно (6а) при значениях ориентационного фактора $M=0.46$ и 0.18 (цифры у кривых), пунктир кривая псевдоупругой деформации монокристалла сплава согласно (3b) при значении ориентационного параметра $m=1$.

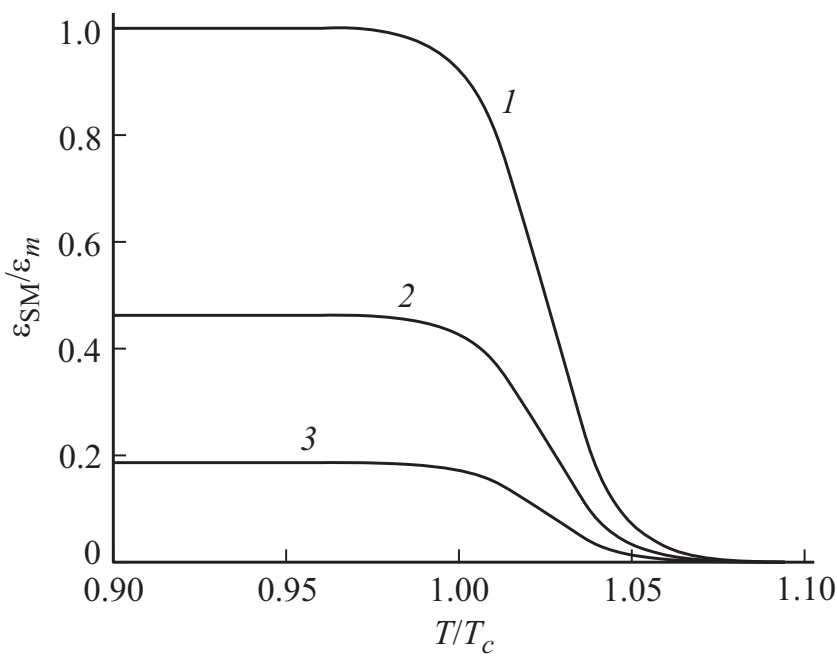

Рис. 4. Зависимость остаточной деформации (деформации памяти формы $\left.\varepsilon_{S M}\right)$ монокристалла сплава с ЭПФ от температуры согласно (8а) (кривая $1, m=1$ ) и поликристалла сплава согласно (8b) при двух значениях ориентационного фактора $M(0.1)=0.46$ (кривая 2) и $M(1)=0.18$ (кривая 3).

указанных выше факторов

$$
\begin{aligned}
\frac{\varepsilon_{S M}}{\varepsilon_{m}} & =\frac{m}{1+\exp \left[\bar{\omega}\left(T / T_{c}-1-\Delta \bar{\sigma}\right)\right]}, \\
\frac{\varepsilon_{S M}}{\varepsilon_{m}} & =\frac{M(s)}{1+\exp \left[\bar{\omega}\left(T / T_{c}-1-\Delta \bar{\sigma}\right)\right]} .
\end{aligned}
$$

На рис. 4 приведены температурные зависимости деформации $\varepsilon_{S M}$ для монокристалла при $m=1$ (кривая 1) и для поликристаллов при $M(0.1)=0.46$ (кривая 2) и $M(1)=0.18$ (кривая 3). Видно, что по сравнению с монокристаллом рост дисперсии зерен по ориентациям существенно снижает деформацию памяти формы поликристалла.

2.2. Нанокристалличность. Мартенситному превращению сплава предшествует предмартенситное состояние и потеря решеткой стабильности. Объем зародышей предмартенсита $\omega_{0}=\pi d_{0}^{2} a$, где $a-$ параметр решетки, определяется корреляционным радиусом $R=d_{0} / 2$. $\mathrm{O}$ его величине можно судить по характерной для предмартенситного состояния наноструктуре поверхности кристалла в виде твида [11] или апельсиновой корки [12]. Дальнейшее снижение температуры приводит к исчезновению пространственной наномодуляции поверхности кристалла и образованию петель дислокаций мартенситного превращения.

Результаты МД-моделирования показывают [3], что в нанокристаллическом сплаве с ЭПФ (TiNi) мартенситная деформация под действием напряжения начинается c образования в центре нанозерен петель дислокаций превращения. Петли, расширяясь, достигают границ зерен. Из-за взаимодействия петель в пересекающихся габитусных плоскостях в нанозернах возникает мартенситная субнаноструктура. Очевидно, что в монокристалле в отсутствие границ зерен и других препятствий для петель превращения они могут расширяться до поверхности кристалла. В нанокристаллическом сплаве размер петель и, следовательно, зародыши мартенсита ограничены размерами зерен $d$. В результате для объема зародыша получаем соотношение $\omega(d)=a d^{2} / 4$ [10]. С учетом этого обстоятельства и (7) зависимость коэффициента деформационного упрочнения $\theta_{m}$ от размера нанозерен определяется уравнением

$$
\theta_{m}(d)=\frac{4 k T}{\omega(d) M(s) \varepsilon_{m}^{2}} \sim 1 / d^{2} .
$$

Рис. 5, $a$ демонстрирует зависимость коэффициента $\theta_{m}$ от размера нанозерен в нанокристаллическом сплаве $\mathrm{TiNi}$, полученную при МД-моделировании сплава (светлые квадратики [3]) и в эксперименте (темные квадратики $[3,4])$. На рис. $5, b$ результаты $[3,4]$ приведены в координатах $\theta_{m}^{-1 / 2}-d$. Видно, что они качественно согласуются с теоретическим соотношением (9). Для количественного согласия требуется, как и в случае с ориентацией зерен, учесть возможную дисперсию размеров зерен в поликристалле.

Для оценки влияния дисперсии на коэффициент $\theta_{m}$ воспользуемся лог-нормальным распределением зерен по размерам,

$$
f(x, d, s)=\frac{1}{s x \sqrt{2 \pi}} \exp \left[-\frac{1}{2}\left(\frac{\ln (x / d)}{s}\right)^{2}\right] .
$$

Усредняя в (9) $1 / d^{2}$ по распределению (10),

$$
\left\langle d^{-2}\right\rangle=\int_{0}^{\infty} x^{-2} f(x, d, s) d x,
$$



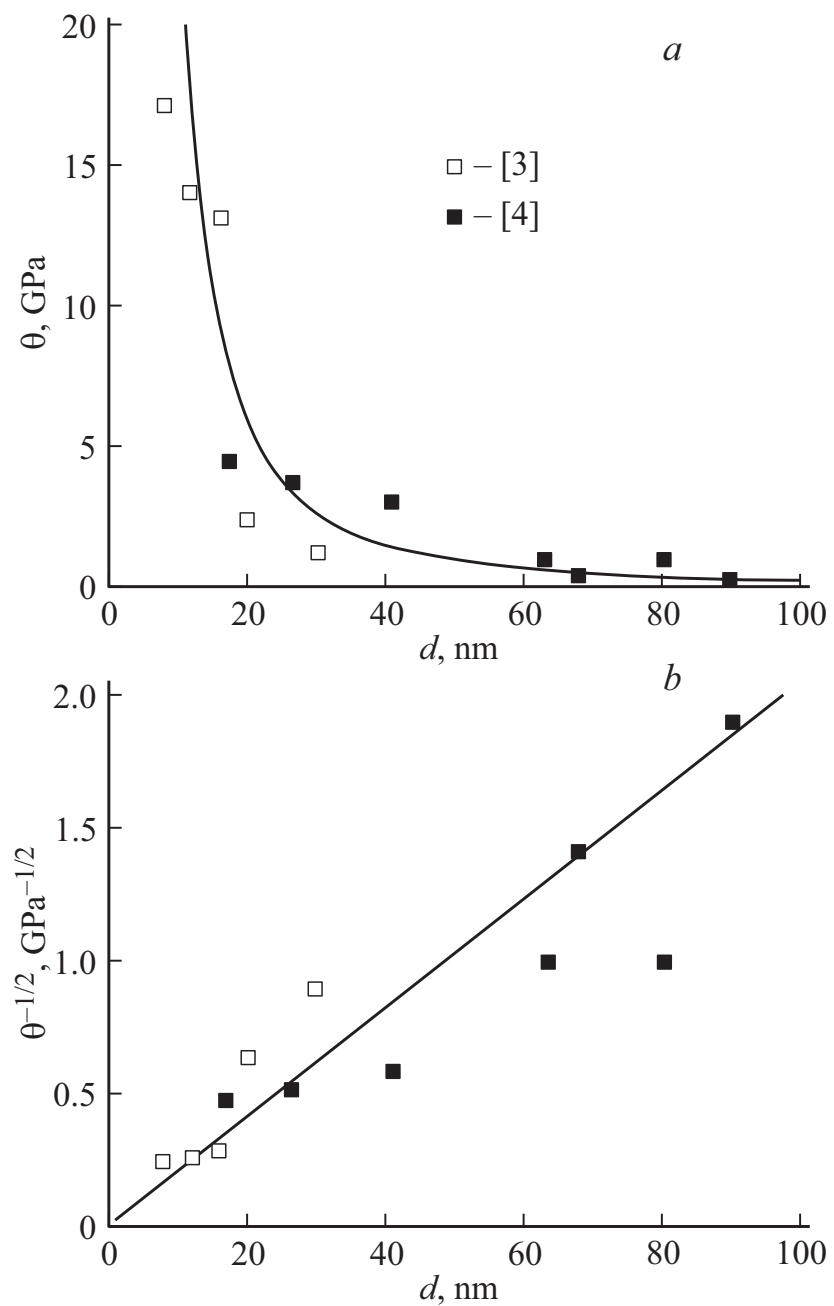

Рис. 5. Зависимость коэффициента деформационного упрочнения нанокристаллического сплава $\mathrm{TiNi}$ от размера нанозерен согласно соотношению (11) в координатах $\theta_{m}-d \quad(a)$ и $\theta_{m}^{-1 / 2}-d \quad(b)$. светлые квадратики результаты МД-моделирования [3], темные - результаты эксперимента [4].

находим, что $\left\langle d^{-2}\right\rangle=d^{-2} \exp \left(2 s^{2}\right)$. В результате, для коэффициента (9) получаем окончательное выражение

$$
\theta_{m}(d)=\frac{4 k T \exp \left(2 s_{2}^{2}\right)}{\omega(d) M\left(s_{1}\right) \varepsilon_{m}^{2}} \sim 1 / d^{2} .
$$

где для ориентационных и размерных дисперсий введены разные обозначения соответственно $s_{1}$ и $s_{2}$. Кривые на рис. 5, $a$ и $b$ построены согласно соотношению (11) при следующих значениях параметров: $T=400 \mathrm{~K}, a=0.4 \mathrm{~nm}$, $\varepsilon_{m}=0.05, M(0.43)=1 / M_{T}=0.33$ и $s_{2}=1.15$. Видно, что наблюдается не только качественное, но и хорошее количественное согласие теории РМП и эксперимента.

\section{3. Заключение}

Теория размытых мартенситных переходов (в более широком аспекте, теория размытых фазовых переходов первого рода [8]) описывает равновесие фаз на макрои мезоуровне, а также влияние различных структурных факторов на это равновесие. Она является инструментом анализа и моделирования мартенситных переходов в сплавах с эффектом памяти формы и их силового и деформационного поведения. Выше было продемонстрировано, как теория РМП позволяет проанализировать влияние поликристалличности и нанокристалличности сплава с ЭПФ на кривую его псевдоупругой деформации и установить зависимость ее параметров от распределения нанозерен по ориентациям и размерам.

\section{Список литературы}

[1] Shape Memory Materials / Eds K. Otsuka, C.M. Wayman. Cambridge University Press, Cambridge (1999). 284 p.

[2] Е.Ю. Панченко, Ю.И. Чумляков, И.В. Киреева, А.В. Овсянников, Х. Сухитоглу, Н. Караман, Г. Майер. ФММ 106, 6, 597 (2008)

[3] W.-S. Ko, S.B. Maisel, B. Grabovsski, J.B. Jeon. Acta Mater. 123, 90 (2017).

[4] A. Ahadi, Q. Sun. Acta Mater. 90, 272 (2015).

[5] T. Waitz, T. Antretter, F.D. Fischer, N.K. Simha, H.P. Karnthaler. J. Mech. Phys. Solids. 55, 419 (2007).

[6] Н.Н. Куранова, Д.В. Гундеров, А.Н. Уксусников, А.В. Лукьянов, Л.И. Юрченко, Е.А. Прокофьев, В.Г. Пушин, Р.3. Валиев. ФММ 108, 6, 589 (2009).

[7] J.M. San Juan, M.R. Nó, Ch.A. Schuh. Adv. Mater. 20, 272 (2008).

[8] Г.А. Малыгин. УФН 171, 187 (2001).

[9] Г.А. Малыгин. ФТТ 42, 1469 (2000).

[10] Г.А. Малыгин. ЖТФ 79, 12, 60 (2009).

[11] A. Zheludev, S.M. Shapiro, P. Wochner, L.E. Tanner. Phys. Rev. B 54, 15045 (1996).

[12] S.A. Wen, A.G. Khachaturyan, J.W. Morris. Met. Trans. A 12, 581 (1988).

Редактор Ю.Э. Китаев 